\title{
Efectos de la Terapia de Interacción Guiada sobre el bienestar de díadas en riesgo psicosocial
}

\author{
Effects of Interaction Guidance Therapy on wellbeing of dyads in psychosocial risk
}

\author{
Esteban Gómez \\ Protectora de la Infancia, Chile \\ $\&$ \\ Magdalena Muñoz \\ Centro de Estudios y Atención al Niño y la Mujer, Chile
}

(Rec: 30 de junio de 2010 / Acep: 15 de noviembre de 2011)

\begin{abstract}
Resumen
Se presentan resultados de un estudio sobre la efectividad de una intervención con video-retroalimentación para familias vulnerables, llamada Terapia de Interacción Guiada (IG). La muestra estuvo compuesta por 19 díadas -figura parental y niños entre 2 y 10 años-, participantes en programas sociales de intervención familiar. Se evaluó el nivel de ajuste conductual y socioemocional en los niños y adultos, mediante un diseño pre-post de grupo único. Se encontró diferencias estadísticamente significativas $(p<0.05)$ con mejorías en los problemas de los niños, y en el bienestar general y sintomatología ansioso-depresiva del adulto, pero no en las variables de estrés contextual ni en las relaciones sociales del adulto; la IG parece funcionar mejor en niños menores de 6 años. Estos hallazgos indican que la IG sería efectiva para mejorar el bienestar y salud mental de díadas en riesgo psicosocial, requiriéndose más investigación para respaldar esta tendencia preliminar.

Palabras clave: Terapia de interacción guiada, video-retroalimentación, salud mental infantil, familias vulnerables, intervención temprana.
\end{abstract}

\begin{abstract}
Outcomes of a study about the effectiveness of an intervention using video feedback for vulnerable families, called Interaction Guidance Therapy (IG), are presented. The sample was composed by 19 dyads -parent and children between 2 and 10 years-, participants in social programs of family intervention. The level of behavior and emotional adjustment of children and adults was assessed, by means of a pre-post design with a single group. Statistical significant differences were found $(p<0.05)$, with improvements in children problems, and in general wellbeing and anxious-depressive symptomatology of the adult, but not in the variables of contextual stress neither in the social relationships of the adult; IG seems to work better with children younger than 6 years of age. These results indicate that IG would be effective to improve the wellbeing and mental health of dyads in psychosocial risk requiring more research to support this preliminary trend. Keywords: Interaction guidance therapy, video-feedback, infant mental health, vulnerable families, early intervention.
\end{abstract}

Correspondencia: Esteban Gómez Muzzio, Unidad de Diseño y Evaluación de Programas, Protectora de la Infancia, Avda. Concha y Toro 1898, Puente Alto, Santiago de Chile, fono: 4848975. E-mail: eagomez@uc.cl.

Los autores desean agradecer las valiosas contribuciones metodológicas de Edgar Valencia al artículo. 


\section{Introducción}

Los programas de intervención familiar pueden centrarse exclusivamente en los padres, en la díada madre-bebé, abordar a la familia en su totalidad e incluso incorporar a la comunidad, pero todos enfrentan el desafío común de fortalecer las competencias de las figuras parentales, mejorar la calidad de las relaciones entre sus miembros, potenciar sus recursos y fortalezas, y facilitar el logro de mejores niveles de calidad de vida y bienestar subjetivo en la familia, según diversos indicadores de resultado, como los niveles de buen trato, salud mental, desarrollo infantil, apego seguro, sensibilidad parental, comunicación o resiliencia familiar, entre otros (Gómez \& Haz, 2008; Gracia, 1997; Weiss \& Jacob, 1989).

Una de las herramientas que en la última década ha mostrado un enorme potencial para el avance técnico en la intervención con familias vulnerables y multiproblemáticas, tanto por su eficacia al obtener resultados como por su eficiencia al hacerlo en menos tiempo, es el uso del registro de secuencias de interacción familiar en video, para su posterior análisis y retroalimentación a la familia (Fukkink, 2008; Gómez, Muñoz \& Santelices, 2008; McDonough, 2000).

Sin embargo, no es el video en sí mismo el centro de este avance técnico, sino que la forma de usarlo como recurso dentro de intervenciones familiares cuidadosamente planificadas, las cuales pueden diseñarse ya sea desde un enfoque psicoeducativo (por ejemplo, mediante consejerías orientadas a la promoción de conductas deseables), como desde un enfoque psicoterapéutico (por ejemplo, mediante una terapia breve orientada a la modificación de patrones relacionales). El video entonces, pasa a ser una herramienta que apoya poderosamente la metodología de trabajo propuesta, en coherencia con los resultados que se busca obtener.

A pesar de los grandes avances teóricos y técnicos que se han conquistado en el campo de la intervención familiar (parental y diádica) mediante el uso de video feedback en países como Suiza, Holanda, Canadá o Estados Unidos, se sabe poco en Chile y Latinoamérica sobre su uso y resultados. Una notable excepción es el riguroso trabajo realizado en la década de los '90 por María Angélica Sepúlveda y su equipo del Centro de Investigaciones para la Infancia y la Familia en Caracas, Venezuela, quienes desarrollaron y evaluaron experimentalmente (en cuatro momentos, con un instrumento de observación) una intervención temprana con psicoeducación, consejería y video feedback en 56 díadas madre-bebé de nivel socioeconómico bajo (Sepúlveda, 1996). Se realizó entre 8 y 12 sesiones, promediando 9 sesiones y obteniendo resultados significativos en el desarrollo cognitivo de los bebés, y el estilo comunicativo y bienestar emocional de las madres, en comparación al grupo control (Sepúlveda, 1996). Este estudio, pionero en Latinoamérica, demostró que es viable y efectivo realizar intervenciones manualizadas con familias vulnerables usando la retroalimentación con apoyo de video, y sentando un precedente para su incorporación en programas comunitarios, psicosociales y clínicos de atención.

Asimismo, destaca el trabajo reciente de Vite, Pérez \& Ruiz (2008), quienes realizaron en México una intervención de entrenamiento conductual con video feedback para 9 díadas madre-niño de nivel socioeconómico bajo, con niños que presentaban problemas conductuales (desobediencia) según era detectado por maestras del preescolar. La edad promedio de los niños fue de 5,2 años, con cinco niñas y cuatro niños, y la edad promedio de las madres fue de 31 años. La intervención tuvo entre 7 y 10 sesiones por caso. Los resultados mostraron un incremento significativo en el índice de sensibilidad materna y una reducción del comportamiento infantil aversivo, lo que muestra que se pueden realizar intervenciones de este tipo no solo con bebés sino también con niños mayores, en población de nivel socioeconómico bajo, y obteniendo resultados significativos en medidas de comportamiento infantil y competencia parental.

A pesar de estos notables aportes, el desarrollo y difusión de intervenciones familiares con video feedback ha sido lento en la región, identificándose pocas experiencias documentadas. Este artículo busca ser un aporte para contribuir al desarrollo de intervenciones que incorporen la retroalimentación de interacciones registradas en video, desde el enfoque de la resiliencia familiar, el apego y la parentalidad positiva para el logro de un adecuado desarrollo infantil en contextos de vulnerabilidad psicosocial (Gómez \& Muñoz, en prensa; Gómez \& Kotliarenco, 2010; Gómez et al., 2008; Rey, 2006).

\section{Evidencia empírica de la efectividad del video- feedback}

En un meta-análisis reciente sobre el uso de videofeedback en programas de intervención familiar (Fukkink, 2008), se incluyeron 29 estudios cubriendo a un total de 1.844 familias. Este meta-análisis es el estudio más ambicioso y riguroso realizado hasta la fecha para sistematizar y sintetizar la evidencia empírica en el área, por lo que se constituye en un referente importante para construir criterios orientadores en el diseño y aplicación de programas e intervenciones con video-feedback. Así, cabe señalar que la mayoría de los programas considerados tuvieron un foco en el comportamiento diádico, aunque se fundamentaban desde diversos marcos teóricos, incluyendo la teoría sistémica, psicoanalítica, del aprendizaje social y/o del apego. La duración promedio de los programas fue de 26,2 semanas o cerca de 6 meses, con una media de 15 sesiones y una duración de cada sesión de 1,5 horas promedio. La duración promedio de los registros usados en las sesiones de retroalimentación fue de 7 minutos y la mayoría de los programas siguieron un patrón de grabación, edición y revisión de las imágenes. Los niños en estos estudios tenían 
una edad promedio de 2,3 años, variando entre 0 y 8 años de edad (Fukkink, 2008).

El meta-análisis realizado por Fukkink (2008) mostró efectos positivos y estadísticamente significativos, de tamaño moderado, en intervenciones que utilizan videoretroalimentación para las tres áreas evaluadas: (a) comportamiento parental, (b) las actitudes de los padres y (c) el desarrollo del niño(a). Así, se identificó que tras este tipo de intervención los padres se tornaron más competentes en la interacción con sus hijos pequeños y experimentaron menos problemas en la interacción, ganando más placer en su rol como padres (Fukkink, 2008). Los programas más breves se mostraron más efectivos en mejorar las competencias parentales que los de duración más prolongada, aunque esta relación no se replicó para el número de sesiones realizadas. Los efectos de la intervención fueron más pequeños para el dominio actitudinal en el nivel parental en comparación con los cambios obtenidos en el nivel conductual; asimismo, los resultados fueron menores en el nivel del niño si los padres pertenecían a grupos de alto riesgo, lo que de acuerdo a Fukkink (2008), hace presumir que las intervenciones familiares con video-feedback son más efectivas en aliviar el estrés parental que está específicamente relacionado con temáticas de crianza y vinculación, pero no en aliviar la carga de otros problemas significativos a nivel familiar como la pobreza o el aislamiento social.

Recientemente, se evaluó mediante un ensayo controlado aleatorio una intervención breve de video-feedback para promover la parentalidad positiva (VIPP), focalizada en la interacción diádica y fundamentada en la teoría del apego (Kalinauskiene, Cekuoliene, Van IJzendoorn, BakermansKranenburg, Juffer \& Kusakovskaja, 2009). Dicho estudio se realizó con una muestra no clínica de 54 madres lituanas de clase media con baja sensibilidad parental y sus bebés. Se encontró un aumento significativo en la respuesta sensible, con un tamaño de efecto grande de acuerdo a Cohen $(d=$ 0,78 ), aun tras controlar la edad materna, el nivel educacional, la depresión, estrés cotidiano, eficacia, género del infante, y afecto negativo y positivo del infante. Sin embargo, no se mejoró la seguridad del apego en los infantes en comparación al grupo de control.

\section{La Terapia de Interacción Guiada}

Dentro de aquellas metodologías que usan el videofeedback para mejorar la calidad de la interacción padrehijo en la familia, se encuentra la Terapia de Interacción Guiada. La Interacción Guiada (IG) consiste en una forma de terapia sistémica breve, cuyo objetivo es reforzar los comportamientos funcionales y modificar los patrones disfuncionales, en especial aquellos directamente relacionados con los síntomas que presenta el niño o niña, patrones que una vez identificados constituyen el foco terapéutico (McDonough, 1995). La IG se usa entonces para ayudar al adulto o adultos que ejercen roles parentales a reconocer y desplegar sus habilidades y competencias, a disfrutar la relación con el niño o niña bajo su cuidado y a desarrollar una mejor comprensión de los comportamientos, necesidades y características del niño en cada etapa del desarrollo infantil (McDonough, 1995, 2000).

En el formato de IG implementado en el presente estudio, se sigue un protocolo relativamente estable, que consiste en un ciclo de 3 fases: (a) sesión de juego con la familia, (b) el terapeuta analiza el video y selecciona secuencias a trabajar, y (c) sesión de retroalimentación, donde se reflexiona junto al adulto significativo y se acuerdan tareas inter-sesión (Suárez, Muñoz, Gómez \& Santelices, 2009). La sesión de juego se divide en dos partes: (1) filmación de una secuencia de juego libre entre el niño y la figura parental, tras una consigna muy breve; (2) una vez finalizado el juego, se lleva a cabo una conversación respecto a la experiencia vivida en el juego. La sesión de retroalimentación se divide a su vez en dos partes: (a) revisión y reflexión guiada respecto a las secuencias elegidas por el terapeuta; y (b) toma de acuerdos respecto a modificaciones interaccionales que se intentarán en la rutina diaria y en la próxima sesión de juego videado (Suárez et al., 2009). Esta secuencia se repite hasta que el foco de trabajo co-construido al inicio, avanza lo suficiente para la familia y el terapeuta; los procesos terapéuticos usualmente duran entre 8-12 sesiones, en un rango entre 2 y 6 meses (McDonough, 2000). Los detalles de la aplicación de la técnica psicoterapéutica se han discutido en una publicación previa, ilustrando su uso a través de un caso clínico (Suárez et al., 2009).

La terapia de Interacción Guiada comparte muchos de los principios de otras formas de terapia familiar sistémica, como la importancia de un balance estructural en la familia; de identificar y modificar coaliciones parentales, tales como la triangulación o la parentalización; de clarificar los roles y relaciones familiares; y de considerar la influencia de la propia infancia de los padres (McDonough, 2000). El supuesto básico de este enfoque es que los niños pueden ser ayudados a través del fortalecimiento de la relación con sus cuidadores primarios y de las habilidades de dichas figuras parentales para "leer" adecuadamente las señales comunicativas de su hijo.

Los procesos clínicos centrales de la IG son: (a) estimular a la familia a definir el problema desde su perspectiva; (b) enfatizar las fortalezas de la familia al mismo tiempo que se reconocen sus vulnerabilidades y limitaciones; (c) generar una instancia no crítica al trabajar con la familia, que le permita abrirse a nuevas formas de crianza; (d) usar un enfoque igualitario y cooperativo; y (e) ofrecer perspectivas alternativas a la familia acerca de la crianza de su hijo, a través de una intervención diseñada en forma personalizada (McDonough, 2000). La alianza de trabajo en la IG es un aspecto crítico y el terapeuta debe esforzarse por conseguirla demostrando su genuino interés por ayudar a la familia; asimismo, el terapeuta debe minimizar su rol de 
autoridad para adoptar una posición que rescata los recursos ya presentes al comenzar en las figuras parentales, en los niños y niñas y en la familia como un todo (McDonough, 2000; Suárez et al., 2009).

\section{Aplicaciones de la IG}

En un estudio preliminar desarrollado en Suiza, se aplicó la Interacción Guiada en una muestra clínica de clase media, encontrándose efectos significativos en mejorar las interacciones de las madres con sus infantes, sus percepciones de sus infantes y la sintomatología (Cramer et al., 1990; Robert-Tissot et al., 1996). En Canadá se desarrolló una versión modificada de la Terapia de Interacción Guiada incluyendo un componente de psicoeducación individual, con resultados positivos en infantes con problemas de alimentación, mejorando significativamente la sensibilidad de las figuras parentales y disminuyendo comportamientos disruptivos en la díada en comparación al grupo de control (Benoit, Madigan, Lecce, Shea \& Goldberg, 2001; Madigan, Hawkins, Goldberg \& Benoit, 2006). Además, en Nueva Orleans, Estados Unidos, se usa en un programa de familias de acogida para apoyar los procesos de reunificación de infantes y niños con sus familias de origen, mejorando los indicadores de protección y buen trato (Larrieu \& Zeanah, 2004). En Chile, la IG se ha usado en un programa de familias de acogida tanto en casos de reunificación con la familia de origen como para apoyar los procesos de acogida familiar en niños y niñas de alto riesgo psicosocial (Suárez et al., 2009). Recientemente se ha incorporado como parte de las metodologías posibles de intervención en los programas de intervención breve "Viviendo en Familia" de la Protectora de la Infancia, dirigidos a la prevención secundaria del maltrato infantil y la negligencia parental.

Este artículo presenta los resultados de un estudio con diseño pre-post de grupo único, sobre los efectos de la terapia IG en familias en riesgo psicosocial, específicamente sobre la sintomatología de los niños y de la figura parental. Las hipótesis fueron: (a) los niños(as) que participen en IG mostrarán una reducción estadísticamente significativa en sus problemas conductuales y socioemocionales; (b) las figuras parentales que participen en IG mostrarán una reducción estadísticamente significativa en su sintomatología.

\section{Método}

\section{Instrumentos}

Cuestionario para la Evaluación de Resultados y Evolución en Psicoterapia, OQ-45.2: es un cuestionario de autoevaluación, que mide resultados en psicoterapia en el adulto, permitiendo tener una medida de ajuste o bienestar socioemocional del adulto (von Bergen \& de la Parra, 2002). La evaluación se hace a través de tres sub-escalas: (a) Sintomatología Ansioso-Depresiva (SD), con 25 ítems como "me siento culpable", "me siento irritado, molesto", "me siento triste"; (b) Relaciones Interpersonales (RI), con 11 ítems como "me llevo bien con otros", "me siento contento con mi matrimonio/pareja"; y (c) Rol o Ajuste Social $(R S)$, con 9 ítems como "me siento presionado (estresado) en el trabajo/escuela", "tengo muchas discusiones en el trabajo/escuela". Estas tres sub-escalas se suman entre sí para una escala total de bienestar socioemocional. Demora en promedio 15 minutos en contestarse. Fue desarrollado en Estados Unidos por Lambert (1996) y adaptado y validado en Chile por von Bergen \& de la Parra (2002), obteniendo una consistencia interna medida con alfa de Cronbach en el OQ total, y las escalas SD, RI y RS de 0,91, 0,89, 0,67 y 0,63 en muestra clínica, y de $0,91,0,88,0,71$ y 0,65 para una muestra no clínica. La validez de constructo mostró una sensibilidad de 0,90 (verdaderos positivos) y de 0,93 (verdaderos negativos) (von Bergen \& De la Parra, 2002). En estudios posteriores ha sido utilizado para evaluar psicopatología y cambio en pacientes de un servicio de psiquiatría, apoyando que el instrumento es sensible y válido (Correa, Florenzano, Rojas, Labra, del Río \& Pastén, 2006).

Inventario de Problemas Conductuales y Socioemocionales, IPCS: para el grupo de niños y niñas entre 3-5 años de edad, es una prueba que permite identificar problemas socioemocionales y conductuales en preescolares. Se compone de 3 secciones: (a) una dirigida a problemas en el niño, (b) otra dirigida a problemas en la madre, y (c) una enfocada en situaciones estresantes de las condiciones de vida del grupo familiar. Fue diseñado en Chile por Rodríguez, Lira, Arancibia \& Bralic (2000), con una muestra clínica de 542 niños y una muestra normal de 309 niños. La confiabilidad test-retest dio cuenta de la estabilidad de los puntajes, con porcentajes de acuerdo superiores a $85 \%$ en todos los ítems; la consistencia interna medida con alfa de Cronbach fue de 0,71 en la sección niño y 0,70 en la sección madre; la validez discriminante del instrumento mostró promedios significativamente superiores en la muestra clínica sobre la normal y el análisis de la varianza mostró que el 91\% de la variabilidad de las puntuaciones en la sub-escala niño se explicó por pertenecer al grupo clínico o al normal. El percentil 85 es el punto de corte entre muestra clínica y normal. El IPCS ha sido usado para estudiar el ajuste infantil en familias intactas y separadas en Chile (Donoso \& Villegas, 2000), en preescolares de centros educativos estatales y particulares en Perú (Suero, Coletti \& Mendoza, 2007) y en seguimiento a niños prematuros en Argentina (Rodríguez, Vivas, Cangelosi \& Schapira, 2008), entre otros. En este estudio solo se usó la escala "Niño" y la escala "Situaciones", ya que la escala "Madre" evalúa aspectos similares al OQ-45.2.

Inventario de Problemas Conductuales y Destrezas Sociales, IPCDS: para el grupo de niños/as entre 6-11 años de edad. Es la adaptación chilena elaborada por Montenegro, Bralic, Edwards, Izquierdo \& Maltes (1983), 
del Child Behavior Check List. Cabe señalar que solo se contó con mediciones pre-intervención con el IPCDS, por cuanto los niños en el rango de edad de 6-10 años no finalizaron la intervención.

Ficha de registro abreviado del proceso clínico: se diseñó una ficha de registro para cada sesión y una ficha de registro abreviado del proceso completo, en la cual cada terapeuta resumió: (a) los criterios para indicar la IG; (b) el foco clínico co-construido que orientó la intervención; (c) los contenidos abordados en las sesiones; (d) los principales logros obtenidos en el proceso según su perspectiva; (e) las principales dificultades enfrentadas; y (f) observaciones a la técnica, al caso o al enfoque.

\section{Procedimiento}

Criterios de inclusión/exclusión en el estudio: se incluyeron niños y niñas entre 2 y 10 años de edad, con condiciones que según el juicio clínico de los profesionales los hiciera candidatos adecuados para una terapia de estas características (estar en "edad de juego", no mostrar agresividad o hiperactividad extrema). No se incluyeron casos en que los padres mostraran retardo mental, severa psicopatología (ej., esquizofrenia), o conducta antisocial grave. Tampoco se incluyeron casos en que se sospechara que el adulto responsable pudiera estar cometiendo maltrato constitutivo de delito o abuso sexual hacia el niño. Como indicación o motivo de consulta, se consideraron todos los casos que mostraran problemas de relación o vinculación afectiva entre la figura parental y el niño(a), como temática principal a abordar. Secundariamente, se podían incluir niños con otros problemas, como dificultades de alimentación, problemas conductuales, entre otros.

Participación libre e informada: todos los casos fueron invitados a participar libremente, explicándoles un documento de consentimiento informado registrado por escrito y triplicado (una copia para el adulto responsable, para el equipo profesional y para los investigadores). Todas las personas tuvieron el derecho a no participar del estudio o a retirarse en cualquier momento; la participación fue voluntaria. Además del consentimiento informado, se firmó un contrato terapéutico que explicaba detalladamente la metodología de la terapia de interacción guiada (juego, grabación en video, análisis de secuencias, etc.). De esta forma, todas las personas invitadas a participar podían rechazar su consentimiento para incorporarse al estudio, pero recibir de todas formas la prestación.

Aplicación de los instrumentos: los instrumentos fueron aplicados por un profesional o técnico distinto del terapeuta encargado de realizar la IG, de tal forma de independizar las mediciones. Los instrumentos se aplicaron en dos ocasiones: (a) antes de iniciar la intervención y (b) al finalizar el tratamiento. La corrección, digitación y análisis de los instrumentos fue realizada por el primer autor, no por los propios terapeutas quienes se mantuvieron ciegos a esta información durante los tratamientos.

\section{Participantes}

La muestra inicial estuvo constituida por 23 niños y sus cuidadores. Sin embargo, tres rechazaron participar del estudio y uno no pudo comenzar por traslado a vivir a otra ciudad. Así, la muestra final estuvo constituida por 19 niños y 19 adultos en situación de riesgo psicosocial, pertenecientes a programas sociales de intervención con familias vulnerables implementados por la Protectora de la Infancia.

La edad promedio de los niños fue 5,3 años ( $D S=2,33)$, entre 2 años y 10 años de edad, con un $63,2 \%$ de niños $(n=12)$, y $36,8 \%$ de niñas $(n=7)$. En el caso de los adultos, la edad promedio fue 38,2 años $(D S=12,36)$, entre un mínimo de 20 años y un máximo de 58 años de edad, con un 15,8\% de hombres $(n=3)$ y un $84,2 \%$ de mujeres $(n=16)$.

Tabla 1: Características de los participantes (n, porcentaje, promedio y desviación estándar).

\begin{tabular}{lccc}
\hline & Hombres & Mujeres & Total \\
\hline Niños & $\mathrm{n}=12$ & $\mathrm{n}=7$ & $\mathrm{n}=19$ \\
Sexo & $63,2 \%$ & $36,8 \%$ & $5,3(2,33)$ \\
Edad & $5,0(2,2)$ & $5,9(2,7)$ & $\mathrm{n}=19$ \\
Adultos & $\mathrm{n}=3$ & $\mathrm{n}=16$ & $38,2(12,36)$ \\
Sexo & $15,8 \%$ & $84,2 \%$ & $36,4(11,9)$ \\
Edad & $47,0(13,2)$ & $\mathrm{n}=6$ & $30,5(6,9)$ \\
Terapeutas & & $66,7 \%$ & $50,0 \%$ \\
Edad & & $7,25(5,68)$ & \\
Profesionales Tituladas & & & \\
Especialización clínica (postítulo/magíster) & & & \\
Años de experiencia & & & \\
\hline
\end{tabular}


Respecto a los terapeutas, se contó con un total de seis terapeutas mujeres, con un promedio de edad de 30,5 $(D S=6,9)$, distribuidas en dos alumnas en práctica y cuatro profesionales, tres de las cuales contaban con diplomado y postítulo/magíster, promediando 7,25 años de experiencia profesional $(D S=5,68)$. Las terapeutas participaron en una capacitación teórico/práctica de un día, se les facilitó artículos sobre el uso de IG y un protocolo que detallaba la implementación del estudio. En total, tuvieron a su cargo 3,2 casos en promedio $(D S=2,6)$; cada alumna trató solo un caso, bajo la supervisión directa de una psicoterapeuta con magíster en psicología clínica y experiencia previa en IG.

\section{Análisis de Datos}

Primero se realizó un análisis comparativo para indagar por diferencias entre quienes finalizaron el proceso de Interacción Guiada y quienes no, utilizando técnicas de estadística descriptiva y pruebas ANOVA y Chi Cuadrado en función de las siguientes variables: edad del niño, edad de la figura parental, bienestar general del adulto y bienestar general del niño al momento de iniciar la terapia, problemas en rango clínico versus rango normal en los adultos y niños; y número de sesiones realizadas.

Posteriormente se realizó una caracterización de la intervención en términos de su duración y número de sesiones, describiendo la situación inicial y final de los casos. Luego se contrastaron las hipótesis del estudio utilizando la prueba $T$ Student para muestras pareadas y se calculó el tamaño de efecto para aquellas variables que mostraron diferencias estadísticamente significativas; adicionalmente, se compararon las variables relaciones interpersonales, rol social y situaciones/condiciones de vida, previas y posteriores a la intervención, mediante las mismas técnicas. Debido a que sólo se tuvo mediciones pre-post en el grupo de niños menores de 6 años, se analizó únicamente el OQ-45.2 y el IPCS. En todas las pruebas se usó como criterio de significancia estadística un alfa de 0,05 .

Por último, se realizó una síntesis de la información recopilada en las fichas de registro abreviado del proceso IG, para identificar los principales temas clínicos que surgieron, agrupándolas en tres grupos de resultados: (a) indicaciones y foco clínico; (b) logros y dificultades percibidas; y (c) observaciones a la técnica.

\section{Resultados}

\section{Procesos terminados versus no terminados}

De los 19 niños(as) y 19 adultos que iniciaron $\mathrm{IG}, 13$ $(68 \%)$ completaron toda la intervención, tres $(16 \%)$ interrumpieron el proceso tras 3-4 sesiones, aduciendo razones laborales que tornaban incompatibles los tiempos y horarios de la intervención propuesta, mientras que tres $(16 \%)$ no adhirieron a la terapia por escasa motivación con el tratamiento, asistiendo solo a 1-2 sesiones. En estos 6 casos no se contó con evaluaciones post.

La duración promedio de los 19 procesos de IG fue de 3,06 meses ( $\mathrm{DS}=1,67)$, con un mínimo de 1 mes (3 semanas) y un máximo de 6 meses (25 semanas). El promedio de sesiones en total fue de $6,9(\mathrm{DS}=4,9)$, con un mínimo de 1 sesión y un máximo de 16 sesiones, observándose diferencias importantes entre quienes terminaron el proceso y quienes no, con un promedio de 9,4 sesiones entre quienes finalizaron ( $\mathrm{DS}=4,1$ ), versus un promedio de 2,5 sesiones entre quienes no finalizaron $(\mathrm{DS}=1,4)$ la intervención, siendo esta diferencia estadísticamente significativa, $F(1$, 15) $=13,994, p=0,002$.

Por otra parte, sólo se obtuvo diferencias significativas en la edad del niño, $F(1,21)=24,446, p=0,000$. No se observaron diferencias en la edad del cuidador, $F(1,16)=$ $0,640, p=0,435$, el grado de bienestar general del cuidador, $F(1,15)=1,089, p=0,313$, la sintomatología y problemas del niño, $F(1,18)=2,917, p=0,105$, ni en el rango clínico versus normal de problemas en niños, $\chi^{2}(1, N=16)=1,667$, $p=0,308$ o adultos, $\chi^{2}(1, N=17)=0,215, p=1,000$, al momento de iniciar los procesos, entre quienes completaron versus quienes no completaron el tratamiento. De esta forma, la "edad del niño" parece ser un factor relevante a considerar en el presente estudio; al analizar los promedios de edad, se observa que los niños que finalizaron promediaron 4,2 años de edad (DS=1,7), mientras que aquellos que no terminaron el proceso promediaron 7,7 años $(\mathrm{DS}=1,6)$. Al observar los datos, el corte que distingue entre quienes terminaron y no terminaron el proceso estuvo en torno a los 6 años de edad.

\section{Indicaciones y foco clínico}

El análisis de la información reportada por los terapeutas en las fichas de registro de proceso, mostró que la IG se indicó frente a: (a) problemas de interacción familiar disfuncional (problemas de vínculo cuidador-niño, adulto no expresa sentimientos al niño, adulto controlador en la interacción, niño temeroso del adulto); (b) problemas en las competencias parentales (disciplina, límites, manejo conductual); y (c) problemas conductuales y socioemocionales en el niño (síntomas ansiosos, excesiva inhibición, dificultades para controlar conducta y expresión de emociones).

Se pudo identificar cinco focos clínicos en los procesos de IG: (a) mejorar la vinculación, acercamiento y expresión afectiva en la díada; (b) disminuir conductas controladoras del adulto; (c) mejorar competencias parentales de manejo conductual; (d) aumentar la sensibilidad del adulto frente a las necesidades socio afectivas del niño/a; y (e) reforzar conductas protectoras del adulto al niño. 
Los contenidos conversados en las sesiones de retroalimentación a la figura parental se resumen en los siguientes: sensibilidad parental, competencias parentales, autonomía y necesidades de los niños, importancia del vínculo afectivo, historia de abandono infantil, distancia versus expresión emocional diádica, comunicación (turnos, escuchar), estimulación cognitiva, regulación de emociones y conductas, y sentido y manejo de la hostilidad infantil.

\section{Sintomatología del niño y del cuidador}

Los análisis descriptivos mostraron que el nivel de la sintomatología de los niños/as tuvo una distribución aproximadamente normal, tanto antes $(Z-K S=0,796 ; p=0,476)$ como después $(Z-K S=0,534 ; p=0,899)$. Esto se repite para el nivel de ajuste socioemocional global del cuidador antes $(Z-K S=0,906 ; p=0,337)$ y después $(Z-K S=0,613 ; p=$ $0,772)$, y para el nivel de sintomatología ansioso/depresiva del cuidador antes $(Z-K S=0,849 ; p=0,409)$ y después $(Z-K S=0,594 ; p=0,808)$, siendo pertinente realizar la prueba $\mathrm{T}$ de Student en la muestra relativamente pequeña de este estudio.

$\mathrm{Al}$ inicio de la intervención, cinco niños se ubicaron en el rango clínico de problemas conductuales y socioemocionales (sobre percentil 85), mientras que posterior a la terapia, todos los niños estaban bajo el rango clínico de problemáticas (igual o menor al percentil 85). Del total de 13 niños con mediciones pre y post, 10 niños disminuyeron sus puntajes mejorando su nivel general de bienestar, un niño mantuvo su estado inicial y dos niños aumentaron sus puntuaciones empeorando su condición, aunque ningún caso pasó de un rango sub-clínico a un rango clínico de problemas conductuales y socioemocionales.

Como se muestra en la Tabla 2, los resultados del contraste de hipótesis mostraron diferencias estadísticamente significativas de reducción en los niveles de problemas conductuales y socioemocionales de los niños después de la intervención en comparación a la línea de base, $t(13)=2,944, p=0,012$, con un tamaño del efecto alto $(d=0.817)$.

Un análisis cualitativo de las puntuaciones mostró que los principales cambios en los niños se reflejaron en la disminución de los niveles de agresividad, el comportamiento extraño, una imagen disminuida y en la presencia de temores en el niño o niña, según fue percibido y reportado por la figura parental en el instrumento.

El contraste de hipótesis para el nivel de ajuste socioemocional general de la figura parental mostró una diferencia estadísticamente significativa, $t(9)=3,055, p=0,014$, con un tamaño de efecto grande $(d=1,038)$. En la escala total del OQ se observó que 7 de los 10 cuidadores disminuyeron sus puntuaciones mejorando su bienestar general, un cuidador mantuvo su situación inicial y 2 cuidadores aumentaron sus puntuaciones mostrando una reducción en su bienestar general, pero sin llegar a rango clínico.

Asimismo, el nivel de sintomatología ansioso/depresiva en la figura parental mostró una reducción estadísticamente significativa, $t(9)=2,906, p=0,017$, con un tamaño de efecto grande $(d=0,947)$. Es decir, posterior a la Interacción Guiada, se observaron mejorías estadísticamente significativas con un tamaño de efecto grande, en el nivel de bienestar general y en la sintomatología ansioso-depresiva de las figuras parentales, las cuales se representan visualmente en el gráfico 1. Un análisis cualitativo mostró que los cambios mayores se registraron en indicadores como sentirse solo, débil y triste, sentirse nervioso e irritado, y en la experimentación de dolores de cabeza de la figura parental.

\section{Contexto familiar y social}

Los análisis descriptivos mostraron que la variable relaciones interpersonales tuvo una distribución aproximadamente normal, tanto antes $(Z-K S=0,624 ; p=0,781)$ como después $(Z-K S=1,001 ; p=0,218)$; igual que para el rol social del cuidador antes $(Z-K S=0,749 ; p=0,574)$ y después $(Z-K S=0,642 ; p=0,735)$; y para las situaciones

Tabla 2: resultados pre-post de la Terapia de Interacción Guiada en díadas en riesgo social.

\begin{tabular}{lcccccc}
\hline \multicolumn{1}{c}{ Indicador } & $\begin{array}{c}\text { Pre }- \text { IG } \\
\text { Media }(D S)\end{array}$ & $\begin{array}{c}\text { Post }- \text { IG } \\
\text { Media }(D S)\end{array}$ & $\begin{array}{c}\text { Cambio } \\
\text { Media }(D S)\end{array}$ & $\begin{array}{c}\text { T-Student } \\
(\text { dos colas) }\end{array}$ & Sig. & Tamaño Efecto \\
\hline IPCS (n=13) & & & & & & \\
Síntomas Niño & $11,31(4,85)$ & $8,31(3,75)$ & $-3,00(3,67)$ & $\mathrm{t}(12)=2,944$ & $\mathrm{p}=0,012^{*}$ & $\mathrm{~d}=0,81$ \\
Situaciones & $7,69(2,81)$ & $6,62(2,63)$ & $-1,07(2,66)$ & $\mathrm{t}(12)=1,460$ & $\mathrm{p}=0,170$ & \\
OQ-45.2 (n=10) & & & & & & \\
Ajuste total & $46,10(15,23)$ & $36,20(21,92)$ & $-9,9(10,24)$ & $\mathrm{t}(9)=3,055$ & $\mathrm{p}=0,014^{*}$ & $\mathrm{~d}=1,03$ \\
Síntomas Adulto & $26,3(9,79)$ & $20,10(13,96)$ & $-6,20(6,74)$ & $\mathrm{t}(9)=2,906$ & $\mathrm{p}=0,017^{*}$ & $\mathrm{~d}=0,94$ \\
Relaciones con otros & $10,40(4,57)$ & $8,00(6,75)$ & $-2,40(3,44)$ & $\mathrm{t}(9)=2,207$ & $\mathrm{p}=0,055$ & \\
Rol Social & $9,40(2,63)$ & $8,10(3,45)$ & $-1,30(2,83)$ & $\mathrm{t}(9)=1,452$ & $\mathrm{p}=0,180$ & \\
\hline
\end{tabular}

$* \mathrm{p}<0.05$. Los tamaños de efecto son grandes según criterios de Cohen.

Nota: * indica un cambio estadísticamente significativo con $p<0.05$. 
de vida de la figura parental antes $(Z-K S=1,179 ; p=0,091)$ y después $(Z-K S=1,027 ; p=0,203)$, mostrando la pertinencia de realizar la prueba $\mathrm{T}$ de Student en las variables descritas.

Aunque se observaron disminuciones en todas las puntuaciones de problemas en el contexto familiar y social, no fueron estadísticamente significativas para ninguna de las sub-escalas consideradas, ni en las relaciones interpersonales, $t(9)=2,207, p=0,055$, ni en el rol social/laboral, $t(9)=1,452, p=0,180$, ni en las situaciones o condiciones de vida del adulto significativo, $t(12)=1,460, p=0,170$.

\section{Logros y dificultades percibidas}

De acuerdo a lo señalado por las terapeutas, el mayor logro que se observó en los procesos de IG fue un mayor disfrute de la interacción, del estar juntos y pasarla bien. Asociado a esto, se observó en muchos casos un aumento en la sensibilidad de la figura parental para visualizar y entender adecuadamente las señales comunicativas y para identificar las necesidades de sus hijos. Secundariamente, se menciona en los registros clínicos una disminución de las conductas controladoras del adulto, incremento de muestras de afecto físico adecuado, y una mejor distinción y ampliación de los límites puestos al hijo. En los niños, las terapeutas refirieron observar un mayor sentido de pertenencia familiar, mayor autonomía y confianza en contextos interpersonales, mejor inserción del niño en su grupo de pares y más muestras de afecto del niño hacia el adulto.

Entre las dificultades señaladas, las terapeutas enfatizaron recurrentemente los problemas contextuales que dificultan una asistencia regular al proceso, problemas de adherencia al programa en general que se traspasan al proceso clínico, la falta de un motivo de consulta inicial en la familia, y la necesidad de realizar intervención en crisis en ciertas sesiones, lo que complicó atenerse al foco trazado.

Finalmente, en cuanto a observaciones que realizaron a la técnica clínica estuvo: (a) realizar grabaciones en la casa de una familia para superar las dificultades de asistencia; (b) incorporar el trasfondo religioso de un adulto para la construcción de la alianza de trabajo; (c) entregar un set de juguetes similares a los usados en la IG, para favorecer la interacción lúdica en el hogar y la transferencia de los aprendizajes. En varias fichas se reiteró la importancia de coordinar el enfoque de trabajo con el resto del equipo, ya que la IG se inserta y articula con otras intervenciones que pueden abordarse desde perspectivas diferentes, dificultando la coherencia del proceso.

\section{Discusión}

El presente estudio evaluó el efecto de la Terapia de Interacción Guiada (McDonough, 1995, 2000; Suárez et al., 2009), sobre niños y adultos de familias en riesgo psicosocial. Tras la intervención, se encontraron diferencias estadísticamente significativas y tamaños de efecto grande según criterios de Cohen, en los problemas conductuales y socioemocionales de los niños y niñas, y en el bienestar general y sintomatología ansioso-depresiva de las figuras parentales. El hallazgo de tamaños de efecto grande reafirma lo reportado en evaluaciones experimentales de intervenciones con video-feedback sobre medidas de sensibilidad parental (Kalinauskiene et al., 2009). En cambio, no se encontraron cambios estadísticamente significativos en las variables de relaciones interpersonales, rol social ni situaciones o condiciones de vida del adulto.

Así, los resultados de este estudio sugieren que la IG puede afectar positivamente el bienestar psicológico de

Gráfico 1: Cambios pre-post Interacción Guiada en familias vulnerables.

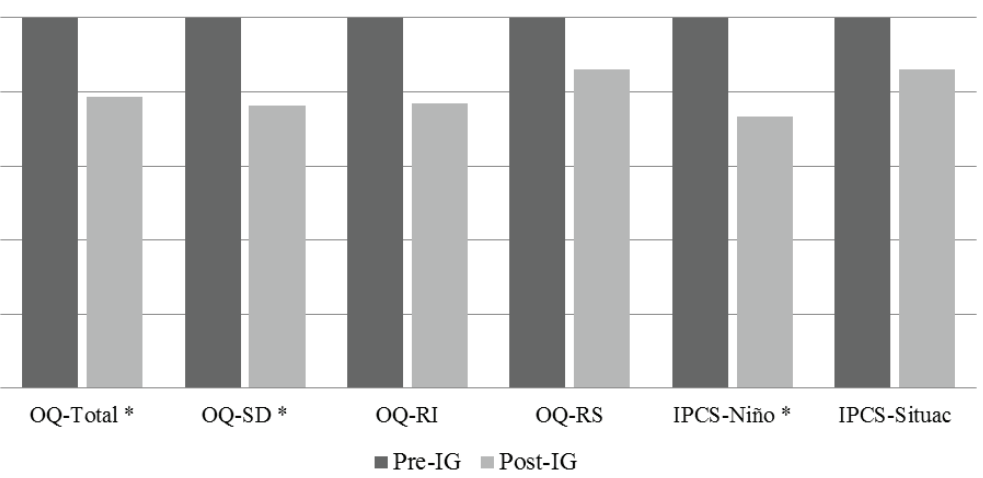

Nota: * indica un cambio estadísticamente significativo con $\mathrm{p}<0.05$. 
la díada, pero no las variables de contexto familiar como la relación de pareja, el aislamiento social, el desempeño laboral o el hacinamiento en el hogar, en línea con las conclusiones del meta-análisis realizado por Fukkink (2008): las intervenciones con video-feedback serían más efectivas en disminuir el estrés parental relacionado específicamente con la crianza de los hijos y con la dinámica relacional, pero no en aliviar el estrés de otros problemas familiares que requerirían otro tipo de intervenciones. Esta idea resulta coherente con la propuesta que hemos hecho en publicaciones previas (Gómez et al., 2008; Suárez et al., 2009), resaltando la importancia de diseñar intervenciones articuladas teórica y metodológicamente para el logro de los desafiantes objetivos que persiguen los programas de intervención familiar. Esto supone un desafío significativo a la competencia del profesional, a su creatividad y compromiso, a su sensibilidad y sabiduría, y a la estructura de soporte técnico, administrativo, financiero e institucional que se requiere (Gómez \& Haz, 2008; Weiss \& Jacob, 1989).

El análisis respecto a los procesos finalizados versus no finalizados, sólo se asoció al número de sesiones realizadas y a la edad del niño, pareciendo funcionar mejor en niños menores de 6 años; eso sugiere que tal vez se requieran otros formatos de tratamiento con niños escolares (Vite et al., 2008), o que la IG requiera ser adaptada para este grupo etario específico. La edad escolar conlleva la influencia de otros contextos sociales además de la familia, lo que puede hacer que un enfoque centrado en la díada no sea suficiente. Por otro lado, ciertas pautas de comunicación y comportamentales ya han sido aprendidas por el niño, consolidadas en la dinámica familiar y por lo tanto pueden requerir de otras herramientas y estrategias para mejorar la dinámica familiar y el manejo conductual de los niños.

Al tratarse de un estudio exploratorio, las limitaciones metodológicas son diversas: la muestra fue relativamente pequeña, no se contó con un grupo de control ni asignación aleatoria de los participantes, lo que restringe la confianza en que los efectos encontrados hayan sido causados por la intervención; se usaron medidas de auto-reporte como indicadores de resultado, las que si bien cuentan con robusta evidencia de su confiabilidad y validez en población chilena, no permiten dar cuenta de indicadores observacionales de la interacción. Esto limita la generalización de los resultados y enfatiza la necesidad de precaución a la hora de replicar esta intervención. En estudios posteriores deben mejorarse estos aspectos (por ejemplo, incluyendo un grupo de comparación, usando sistemas de observación de los videos), para lograr mayores niveles de respaldo a la efectividad de la Terapia de Interacción Guiada en familias vulnerables.

A pesar de estas limitaciones, este estudio reviste una importancia significativa, al constituirse en evidencia preliminar positiva que promueve avanzar en el diseño e implementación de intervenciones terapéuticas familiares que utilicen video-feedback en Chile. Solo se encuentran disponibles dos experiencias similares: en Venezuela (Sepúlveda, 1996) y en México (Vite et al., 2008), siendo el presente estudio el primero en Latinoamérica en utilizar el enfoque de Terapia de Interacción Guiada de Susan McDonough (2000).

Los resultados cualitativos reportados por las terapeutas, enfatizan que el mayor cambio observable en las sesiones es el aumento progresivo del disfrute en la interacción, iluminando el efecto terapéutico que puede tener introducir aspectos lúdicos en la relación en familias que precisamente sorprenden al declarar que "jamás habíamos jugado juntos antes de hoy" (Suárez et al., 2009). Otro aspecto importante parece ser el aumento en los niveles de sensibilidad parental para identificar, comprender y responder de mejor forma a las señales comunicativas de los hijos e hijas; así, el constructo "sensibilidad parental" tendría un alcance clínico más amplio que solamente la intervención temprana con infantes como ha sido usado hasta ahora (BakermansKranenburg, Van IJzendoorn \& Juffer, 2003). Al mostrar su significativa utilidad en intervenciones clínicas, tanto con infantes como con niños y niñas preescolares, se ha sugerido considerarlo como un proceso básico de todas las prácticas parentales efectivas (Vite et al., 2008; Gómez \& Muñoz, en prensa). Disfrutar el estar juntos, reaprender a leer e interpretar la interacción, practicar nuevas formas de respuesta... Para padres y madres de familias abrumadas por múltiples problemas y factores de estrés, cargados con la culpa y la desilusión de fallar en diversos aspectos de la crianza de sus hijos, este enfoque puede ser un poderoso mensaje de aliento y esperanza que los anime a probar una nueva forma de hacer las cosas.

\section{Referencias}

Bakermans-Kranenburg, M.J., Van IJzendoorn, M.H., \& Juffer, F (2003). Less is more: Meta-analyses of sensitivity and attachment interventions in early childhood. Psychological Bulletin, 129, 195-215.

Benoit, B., Madigan, S., Lecce, S., Shea, B., \& Goldberg, S. (2001). Atypical maternal behavior before and after intervention. Infant Mental Health Journal, 22, 611-626.

Correa, J., Florenzano, R., Rojas, P., Labra, J., del Río, V., \& Pastén, J. (2006). El uso del cuestionario OQ-45.2 como indicador de psicopatología y de mejoría en pacientes psiquiátricos hospitalizados. Revista Chilena de Neuro-Psiquiatría, 44, 258-262.

Cramer, B., Robert-Tissot, C., Stern, D., Serpa-Rusconi, S., Demuralt, M., Besson, G., et al., (1990). Outcome evaluation in brief mother-infant psychotherapy: A preliminary report. Infant Mental Health Journal, $11,278-300$.

Donoso, T., \& Villegas, M.I. (2000). Percepción materna del ajuste socioemocional de sus hijos preescolares: Estudio descriptivo y comparativo de familias separadas e intactas con alto y bajo nivel de ajuste marital. Revista de Psicología, 9, 1-17.

Fukkink, R. (2008). Video feedback in widescreen: A meta-analysis of family programs. Clinical Psychology Review, 28, 904-916.

Gómez, E. \& Kotliarenco, M.A. (2010). Resiliencia Familiar: un enfoque de investigación e intervención con familias multiproblemáticas. Revista de Psicología, 19(2), 103-131. 
Gómez, E. \& Muñoz, M. (en prensa). Trabajando habilidades parentales con familias altamente vulnerables. Revista de Psiquiatría y Salud Mental.

Gómez, E., \& Haz, A.M. (2008). Intervención familiar preventiva en programas colaboradores del SENAME: La perspectiva del profesional. Psykhé, 17, 53-65.

Gómez, E., Muñoz, M., \& Santelices, M.P. (2008). Efectividad de las intervenciones en apego con infancia vulnerada y en riesgo social: Un desafío prioritario para Chile. Terapia Psicológica, 26, 241-251.

Gracia, E. (1997). El apoyo social en la intervención comunitaria. Barcelona: Paidós.

Kalinauskiene, L., Cekuoliene, D., Van IJzendoorn, M.H., BakermansKranenburg, M.J., Juffer, F., \& Kusakovskaja, I. (2009). Supporting insensitive mothers: The Vilnius randomized control trial of videofeedback intervention to promote maternal sensitivity and infant attachment security. Child: Care, Health and Development, 35, 613-623.

Lambert, M., Hansen, N., Umphress, V., Lunnen, K., Okiishi, J., Burlingame, et al., (1996). Administration and scoring manual for the Outcome Questionaire (OQ-45.2). Wilmington, DE: American Professional Credentialing Service LLC.

Larrieu, J. \& Zeanah, C. (2004). Treating Parent-Infant Relationships in the Context of Maltreatment: An Integrated Systems Approach. pp. 243-264. En: Sameroff, A. J., McDonough, S. C. \& Rosenblum, K. L. (Eds) (2004). Treating Parent-Infant Relationship Problems: Strategies for Intervention. New York: The Guilford Press.

Madigan, S., Hawkins, E., Goldberg, S., \& Benoit, D. (2006).Reduction of disrupted caregiver behavior using Modified Interaction Guidance. Infant Mental Health Journal, 27, 509-527.

McDonough, S. (1995). Promoting positive early parent-infant relationships trough interaction guidance. Child and Adolescent Psychiatric Clinics of North America, 4, 661-672.

McDonough, S. (2000). Interaction Guidance: An approach for difficultto-engage families. In C.H. Zeanah (Ed.), Handbook of Infant Mental Health (pp. 485-493). New York: Guildford Press.

Montenegro, H., Bralic, S., Edwards, M., Izquierdo, T., \& Maltes, S. (1983). Salud mental del escolar. Estandarización del Inventario de Problemas Conductuales y Destrezas Sociales de T. Achenbach en niños de 6 a 11 años. Santiago de Chile: CEDEP-UNICEF.
Rey, C. (2006). Entrenamiento de padres: Una revisión de sus principales componentes y aplicaciones. Revista Infancia, Adolescencia y Familia, $1,61-84$.

Robert-Tissot, C., Cramer, B., Stern, D., Rusconi-Serpa, S., Bachmann, J.P., Palacio-Espasa, F., et al., (1996). Outcome evaluation in brief mother-infant psychotherapies: Report on 75 cases. Infant Mental Health Journal, 17, 97-114.

Rodríguez, G., Vivas, S., Cangelosi, M., \& Schapira, I. (2008). Avatares en prematuros de 3 a 5 años y sus madres. Revista del Hospital Materno Infantil Ramón Sardá, 27, 152-154.

Rodríguez, S., Lira, M.I., Arancibia, V., \& Bralic, S. (2000). Inventario de Problemas Conductuales y Socioemocionales para niños entre $3 y$ 5 años. Santiago de Chile: Pontificia Universidad Católica de Chile.

Sepúlveda, M.A. (1996). Proyecto Interacción Temprana Madre-Bebé: Informe técnico final. $\mathrm{N}^{\circ}$ Dossier: 91-0137. Universidad Metropolitana, Caracas: Centro de Investigaciones para la Infancia y la Familia.

Suárez, N., Muñoz, M., Gómez, E., \& Santelices, M.P. (2009). Terapia de interacción guiada: una nueva modalidad de intervención con familias multiproblemáticas y en riesgo social. Terapia Psicológica, 27, 203-213.

Suero, E., Coletti, G., \& Mendoza, J. (2007). Problemas conductuales y socioemocionales en niños de 3 a 5 años en centros educativos iniciales de la ciudad de Huánuco. Investigación Valdizana, 1,103-106.

Vite, A., Pérez, I., \& Ruiz, M. (2008). El impacto de la sensibilidad materna y el entrenamiento a padres en niños con problemas de conducta. Revista Mexicana de Análisis de la Conducta, 34, 165-177.

Von Bergen, A., \& De la Parra, G. (2002). OQ-45.2, cuestionario para evaluación de resultados y evolución en psicoterapia: Adaptación, validación e indicaciones para su aplicación e interpretación. Terapia Psicológica, 20, 161-176.

Weiss, H., \& Jacobs, F. (1989). Evaluating family programs. New York: Aldine de Gruyter. 